\title{
Quantitative Microbial Risk Assessment Applied to Legionella Contamination on Long-Distance Public Transport
}

\author{
Ileana Federigi ${ }^{1, *(1)}$, Osvalda De Giglio ${ }^{2}\left(\mathbb{D}\right.$, Giusy Diella $^{2}$, Francesco Triggiano ${ }^{2}$, Francesca Apollonio ${ }^{2}$, \\ Marilena D'Ambrosio ${ }^{2}$, Lorenzo Cioni ${ }^{3}\left(\mathbb{D}\right.$, Marco Verani ${ }^{1}\left(\mathbb{D}\right.$, Maria Teresa Montagna ${ }^{4}(\mathbb{D})$ \\ and Annalaura Carducci ${ }^{1}$ (D)
}

1 Laboratory of Hygiene and Environmental Virology, Department of Biology, University of Pisa, Via S. Zeno 35/39, 56127 Pisa, Italy; marco.verani@unipi.it (M.V.); annalaura.carducci@unipi.it (A.C.)

2 Department of Biomedical Science and Human Oncology, University of Bari Aldo Moro, Piazza G. Cesare 11, 70124 Bari, Italy; osvalda.degiglio@uniba.it (O.D.G.); giusy.diella@uniba.it (G.D.);

francesco.triggiano@uniba.it (F.T.); francesca.apollonio@uniba.it (F.A.); marilena.dambrosio@uniba.it (M.D.)

3 Scuola Normale Superiore, Piazza dei Cavalieri 7, 56126 Pisa, Italy; lorenzo.cioni@sns.it

4 Regional Reference Laboratory of Clinical and Environmental Surveillance of Legionellosis, Department of Biomedical Science and Human Oncology, University of Bari Aldo Moro, Piazza G. Cesare 11, 70124 Bari, Italy; mariateresa.montagna@uniba.it

* Correspondence: ileana.federigi@unipi.it; Tel.: +39-050-2213646

\section{check for}

updates

Citation: Federigi, I.; De Giglio, O.; Diella, G.; Triggiano, F.; Apollonio, F.; D'Ambrosio, M.; Cioni, L.; Verani, M.; Montagna, M.T.; Carducci, A. Quantitative Microbial Risk Assessment Applied to Legionella Contamination on Long-Distance Public Transport. Int. J. Environ. Res. Public Health 2022, 19, 1960. https:// doi.org/10.3390/ijerph19041960

Academic Editor: Paul B. Tchounwou

Received: 13 January 2022

Accepted: 9 February 2022

Published: 10 February 2022

Publisher's Note: MDPI stays neutral with regard to jurisdictional claims in published maps and institutional affiliations.

Copyright: (C) 2022 by the authors. Licensee MDPI, Basel, Switzerland. This article is an open access article distributed under the terms and conditions of the Creative Commons Attribution (CC BY) license (https:// creativecommons.org/licenses/by/ $4.0 /$ )

\begin{abstract}
The quantitative microbial risk assessment (QMRA) framework is used for assessing health risk coming from pathogens in the environment. In this paper, we used QMRA to evaluate the infection risk of L. pneumophila attributable to sink usage in a toilet cabin on Italian long-distance public transportation (LDT). LDT has water distribution systems with risk points for Legionella proliferation, as well as premise plumbing for drinking water, but they are not considered for risk assessment. Monitoring data revealed that approximately 55\% of water samples $(217 / 398)$ were positive for L. pneumophila, and the most frequently isolated was L. pneumophila sg1 (64\%, 139/217); therefore, such data were fitted to the best probability distribution function to be used as a stochastic variable in the QMRA model. Then, a sink-specific aerosolization ratio was applied to calculate the inhaled dose, also considering inhalation rate and exposure time, which were used as stochastic parameters based on literature data. At L. pneumophila sg1 concentration $\leq 100 \mathrm{CFU} / \mathrm{L}$, health risk was approximately 1 infection per 1 million exposures, with an increase of up to 5 infections per 10,000 exposures when the concentrations were $\geq 10,000$ CFU/L. Our QMRA results showed a low Legionella infection risk from faucets on LDT; however, it deserves consideration since LDT can be used by people highly susceptible for the development of a severe form of the disease, owing to their immunological status or other predisposing factors. Further investigations could also evaluate Legionella-laden aerosols from toilet flushing.
\end{abstract}

Keywords: Legionella pneumophila; long-distance public transport; train; monitoring; bioaerosol; water distribution system; premise plumbing; train; risk assessment; public health

\section{Introduction}

Built environments for residential, tourist accommodation, healthcare, and longdistance public transportation (LDT) are equipped with various types of water storage and distribution systems for hygienic purposes and safe removal of human waste [1]. Apart from their design, the moist engineered surfaces of pipes and water tanks can be sensitive to biofilm growth; thus, they represent an ecological niche for environmental bacteria, which can behave as opportunistic pathogens, such as nontuberculous mycobacteria, Pseudomonas spp., and Legionella spp. [2,3]. Particular attention is dedicated to Legionella spp. since Legionella pneumophila is one of the main etiological agents of epidemic pneumonia associated with water systems throughout the world [4-6]. Many environmental studies 
isolated L. pneumophila in water from premise plumbing, which refers to all piping located downstream of the main water distribution system and within buildings, for example collecting water samples from hot/cold mixing valves in various settings, such as healthcare facilities [7,8], hotels [9,10], cruise ships [11,12], retirement homes [13], private homes, or office buildings [14-16]. Moreover, some studies highlighted the growth of L. pneumophila at the level of the outlets of water systems, such as showers, faucets, and toilet flushing [17]. From these points, a Legionella-laden aerosol can be produced, causing people exposure by inhalation. Therefore, Legionella is a biological hazard also in drinking water systems, as highlighted by the recently released directive on the quality of water intended for human consumption (Directive EU 2020/2184). The new directive recognizes the high health burden of Legionella attributable to inhalation during domestic water uses; thus, it indicates Legionella monitoring in the context of risk assessment of domestic distribution systems.

To prevent sporadic or epidemic Legionellosis cases, guidelines for water safety plans are available for premise plumbing systems either in tourist accommodation, in hospitals, and other living and working settings. Such guidelines are based on a careful study of the water system to design and implement control measures, including the continuous monitoring of Legionella concentrations in water, to evidence the exceeding of the thresholds corresponding to levels of water management actions [18,19]. Such Legionella concentration criteria are generally based on professional judgment and could benefit from a risk-based approach to understand health risk corresponding to different indoor fixture use scenarios [20]. The quantitative microbial risk assessment (QMRA) framework is suitable for such a purpose since it allows the calculation of health risk starting from pathogens monitored (or inferred) in the environment [21]. Overall, the attention on Legionella proliferation is focused on some priority premises, such as healthcare facilities and spa pools [19], but such bacteria can proliferate in all human-made building water systems, including long-distance transportation (LDT), since they are equipped with plumbing and toilet water tanks. On passenger trains, the water for washbasin and toilets is stored in water tanks (from 200 to $1800 \mathrm{~L}$ ) located under the car's roof, and it flows into the toilets by gravity. Tanks are refilled at stations with drinking water supply, but this requirement cannot be fully insured owing to the characteristics of hydraulic systems onboard; therefore, water is not considered suitable for human consumption. Although such water is not ingested, it can be aerosolized during the usage of sinks for handwashing, and it can create an exposure scenario to Legionella. LDT is rarely monitored for Legionella contamination [22] and, consequently, possible infection or illness risk has not yet been explored. To fill this gap of knowledge, the aims of this paper were: (i) to investigate Legionella contamination on LDT through the analysis of 6-year monitoring data from the faucet of toilet cabins and (ii) to develop a QMRA model for sink usage on LDT using monitoring data to understand the probability of Legionella infection in such exposure scenario. Although aerosols produced by toilet flushing could also contribute to the probability of infection, such a scenario has not been modeled owing to the lack of monitoring data. Moreover, we estimated the infection risk corresponding to different Legionella concentration levels in water commonly used for the adoption of control measures.

\section{Materials and Methods}

\subsection{Water Sampling and Legionella Analysis}

In a period of 6 years, periodical monitoring has been carried out from faucets on Italian passenger LDT, with a total of 398 samples from 2012 to 2018 (except for 2016). Microbial detection of Legionella was performed according to the Italian guidelines and as previously described [9]. Briefly, 1 L samples were collected in sterile bottles with sodium thiosulphate $(0.01 \%, w / v)$ to neutralize residual chlorine in the toilet water supplies. Each sample was filtered through $0.2 \mu \mathrm{m}$ pore-diameter polycarbonate membranes, with the membrane then resuspended in $10 \mathrm{~mL}$ of the same water sample and vortexed. A $5 \mathrm{~mL}$ aliquot of the suspension was heat-treated by incubation at $50{ }^{\circ} \mathrm{C}$ for $30 \mathrm{~min}$. Aliquots $(100 \mu \mathrm{L})$ of both the heat-treated and untreated samples were seeded onto glycine van- 
comycin polymyxin cycloheximide (GVPC)-selective medium and incubated at $36 \pm 1{ }^{\circ} \mathrm{C}$ for 10 days in a modified atmosphere $\left(2.5 \% \mathrm{CO}_{2}\right)$. Putative Legionella colonies were subcultured on buffered charcoal yeast extract (BCYE) agar and BCYE agar without cysteine. Colonies that grew only in the presence of cysteine were identified as Legionella. Then, these colonies were serotyped using a latex agglutination test (Biolife Italiana Srl, Milan, Italy) to identify L. pneumophila sg1, L. pneumophila sg2-14, and other species of Legionella spp. Water samples containing <100 CFU per liter (CFU/L) were considered negative for Legionella.

\subsection{Statistical Analysis}

Legionella data were log-transformed before performing statistical analysis to calculate geometric mean and standard deviation. Legionella contamination, separately for each serogroup and total, was also described in terms of the interquartile range (IQR), considering the first and third quartiles of concentration data. To analyze the annual differences in Legionella contamination, a one-way analysis of variance (ANOVA) was performed (results were considered statistically significant when $p<0.05)$. Legionella serogroup loads were categorized into four classes according to Italian water quality guidance for Legionella spp. control and prevention in the plumbing system for potable water distribution: $\leq 100 \mathrm{CFU} / \mathrm{L}$, 101-1000 CFU/L, 1001-10,000 CFU/L, and $\geq 10,001$ CFU/L [23]. All the figures were generated with the R program [24]. Boxplot graphs with individual monitoring data were created using the ggplot 2 package. In each graph, the whiskers represent the minimum and maximum values and the boxes from the $1^{\circ}$ and $3^{\circ}$ quartiles of the dataset. We performed the fitting distributions to the monitoring data of L. pneumophila sg1 (hereafter empirical data). The best-fitting probability distribution was selected from three theoretical distributions (Lognormal, Weibull, Gamma), which are commonly used to approximate microbiological data [25]. The fit of theoretical distributions to empirical data was tested by maximum likelihood estimation (MLE), and the quality of the fit was assessed using the Akaike (AIC) criterion. The analysis was performed in the R program with the fitdistrplus package [24,25], and the quality of the fit was also shown by goodness-of-fit graphs [26].

\subsection{QMRA Methodology}

QMRA is a formal four-step process that uses the environmental concentration of pathogens and the amount of exposure to an environmental matrix (dose) as inputs and then estimates the associated probability (risk) of an adverse outcome (infection or illness) as an output, using pathogen-specific mathematical functions describing the dose-response relationship [21,27] (Figure 1). Although many different Legionella species and strains of $L$. pneumophila can be found in the considered water, for the QMRA model, we chose to focus on L. pneumophila sg1, owing to its epidemiological relevance and abundance in environmental samples and the availability of a published dose-response relationship. Moreover, we considered the infection an endpoint for the risk estimate because the probability of illness following infection is strictly related to the host susceptibility, determined by age, sex, smoking habits, other diseases, and so on [28].

\subsubsection{Exposure Assessment}

The dose was calculated according to the partitioning coefficient approach [29], in which the concentrations of L. pneumophila in water were converted into concentrations in the air using an aerosolization ratio (partitioning coefficient $(P C)$ ) specific for sink exposure. Therefore, the inhaled dose $(D)$ was calculated according to Equation (1), and the values used for each parameter of the exposure assessment $\left(C_{\text {water }}, P C, F_{1-8}, I R, E T\right)$ are shown in Table 1.

$$
D=C_{\text {water }} * P C^{*} F_{1-8} * I R * E T
$$

where $D$ is the dose of L. pneumophila sg1 deposited in the lungs during sink use (number of L. pneumophila sg1), $C_{\text {water }}$ is the concentration of L. pneumophila sg1 in water collected from the sink (CFU/L), PC is the bacterial water to air partitioning coefficient (CFU L/CFU m³), $F_{1-8}$ is the fraction of aerosols in the respirable diameter (between $1 \mu \mathrm{m}$ and $8 \mu \mathrm{m}$ ) produced 
by a sink (\%), IR is the inhalation rate of air breathed during sink use, and ET is the exposure duration in the toilet cabin during and after sink use (min).

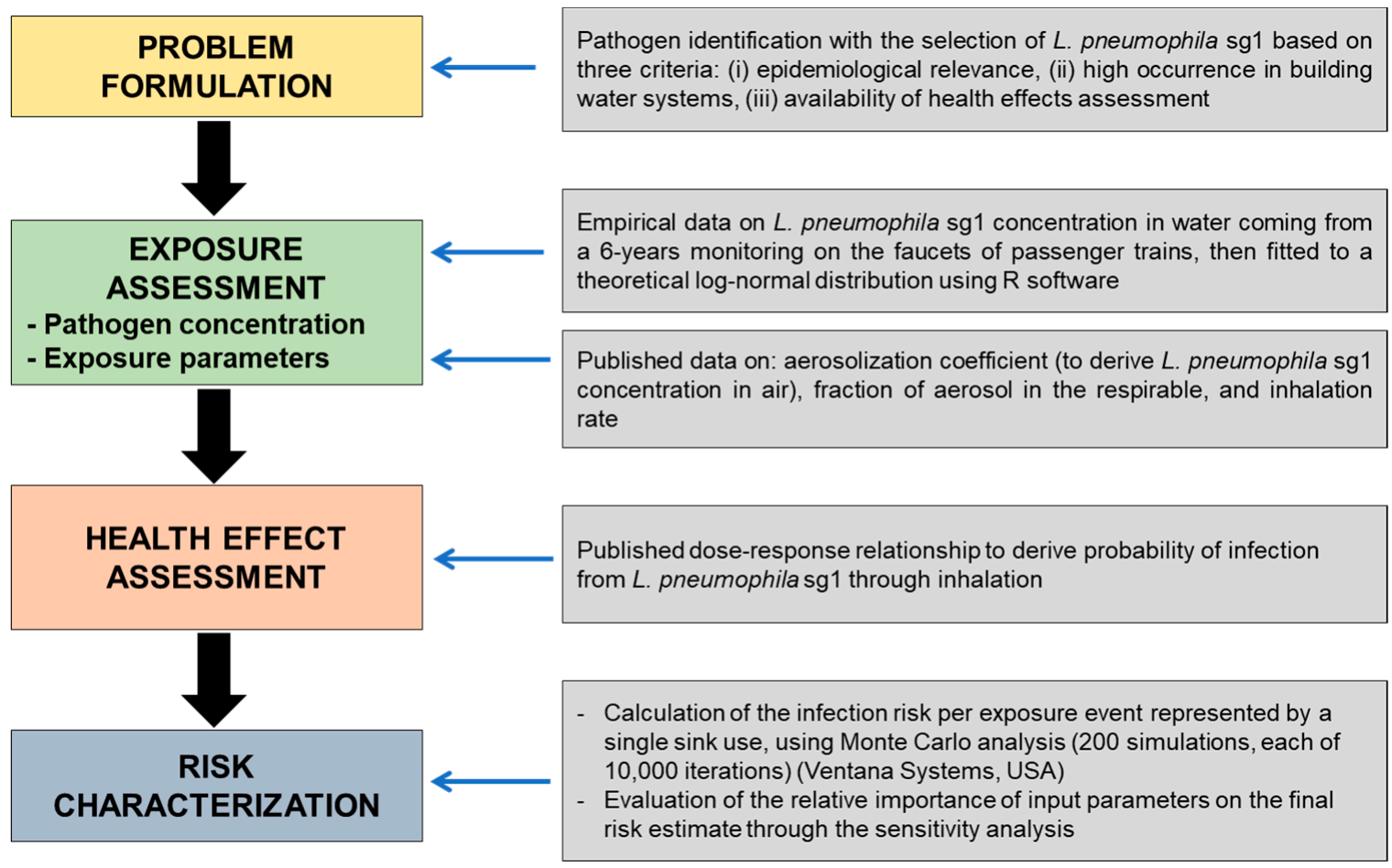

Figure 1. Schematics of the QMRA model framework and model input parameters for the modeled exposure scenario of sink usage on LDT.

Table 1. Distributions and parameters of the exposure assessment in the QMRA framework.

\begin{tabular}{|c|c|c|c|c|}
\hline Input Variables & Description & Unit & Characterization & Source and Comments \\
\hline$C_{\text {water }}$ & $\begin{array}{l}\text { L. pneumophila sg1 } \\
\text { concentration in water }\end{array}$ & CFU/L & $\begin{array}{l}\text { lognormal distribution }^{1} \\
(\mu=8.166848, \sigma=1.521021)\end{array}$ & $\begin{array}{c}\text { This article, based on a } 6 \text {-year } \\
\text { monitoring period }\end{array}$ \\
\hline$P C$ & $\begin{array}{l}\text { Sink partitioning } \\
\text { coefficient }\end{array}$ & $\mathrm{L} / \mathrm{m}^{3}$ & $\begin{array}{c}\text { lognormal distribution }{ }^{1} \\
(\mu=-13.3, \sigma=3.49) \text { truncated on } \\
\text { the interval } \\
{\left[0,2.35 \times 10^{-3}\right]}\end{array}$ & $\begin{array}{l}\text { Hamilton et al., } 2019 \text { [20]. Data } \\
\text { analysis of } 19 \text { paired water and air } \\
\text { samples from hot-water faucets }\end{array}$ \\
\hline$F_{1-8}$ & $\begin{array}{l}\text { Percentage of aerosols in } \\
\text { respirable range (between } \\
1 \text { and } 8 \mu \text { m reported) for } \\
\text { partitioning coefficient }\end{array}$ & $\%$ & point estimate (50) & $\begin{array}{l}\text { Bollin et al., } 1985 \text { [30]. Monitoring of } \\
\text { air samples from hot-water faucets, } \\
\text { and approximately half of recovered } \\
\text { Legionella were between } 1 \text { and } \\
\qquad 8 \mathrm{~mm} \text { aerosol }\end{array}$ \\
\hline$I R$ & Inhalation rate & $\mathrm{m}^{3} / \mathrm{min}$ & $\begin{array}{l}\text { uniform distribution }(\min =0.013 \\
\max =0.017)\end{array}$ & $\begin{array}{l}\text { USEPA } 2011 \text { [31]. Inhalation rate for } \\
\text { individuals engaging in light activities }\end{array}$ \\
\hline$E T$ & Sink use duration & $\min$ & $\begin{array}{l}\text { uniform distribution }(\min =0.5 \\
\qquad \max =1.5)\end{array}$ & $\begin{array}{l}\text { An assumption on the duration of an } \\
\text { individual would stay in the toilet for } \\
\text { hand washing. }\end{array}$ \\
\hline
\end{tabular}

${ }^{1}$ The lognormal distribution of each variable $Y$ has been evaluated as $\exp \left(\mu+\sigma^{*} Z\right)$ where $Z$ is a standardized normal variable with mean 0 and standard deviation 1 , and $\mu$ and $\sigma$ are, respectively, the mean and the standard deviation of a generic normal distribution.

\subsubsection{Dose-Response Assessment}

The dose-response relationship for L. pneumophila among humans has not yet been developed; therefore, data refer to L. pneumophila exposure experiments in an animal model, the guinea pigs, which showed alveolar deposition of aerosol particles comparable with humans and with a similar disease course. The probability of infection of L. pneumophila was therefore calculated using the following exponential dose-response model (Equation (2)):

$$
P_{\text {inf }}=1-\mathrm{e}^{-D^{*} r}
$$


where $P_{\text {inf }}$ is the probability of infection during a single sink use, $D$ is the inhaled dose of $L$. pneumophila derived from the exposure assessment, and $r$ is the probability of one cell to survive the host barriers and successfully initiate the infection, corresponding to 0.06 for $L$. pneumophila [32].

\subsubsection{Risk Characterization and Sensitivity Analysis}

The probability of infection owing to inhalation of L. pneumophila sg1 was computed using Monte Carlo analysis (Vensim package, Ventana Systems, Inc., Harvard, MA, USA) to capture the variability of input parameters modeled as probability distribution functions: L. pneumophila concentration in water, sink partitioning coefficient, and inhalation rate (see Table 1). The Monte Carlo analysis was run for 200 simulations, each one with a random sampling of 10,000 iterations from input parameters of Table 1 varying at random according to their distributions. The final result can be seen as the probability of L. pneumophila infection based on 200 independent measures to improve the accuracy of the health risk analysis. The sensitivity analysis was carried out to test the relative importance of the stochastic variables on the model output (infection risk), and it was performed according to the procedure previously described by Federigi et al. (2020) [33]. Briefly, each of the input parameters was varied, once at a time, according to its own probability distribution function (see Table 1) while keeping each of the other input parameters fixed. In particular, parameters modeled as symmetric probability distributions (inhalation rate and sink use duration) were fixed at the average value, while for other distributions (L. pneumophila sg1 concentration in water, sink partitioning coefficient), modal values were chosen. Then, five simulations were run: one with all the input parameters held at their fixed values and the other four letting the variation of only one parameter at a time. In this way, six arrays of 10,000 values of the $P_{\text {inf }}$ were obtained. Finally, the relative importance of each input parameter was calculated as the average $P_{\text {inf }}$ value of the pairwise differences (in absolute value) between the simulation with all the input parameters at a constant value and the simulation with that parameter varying.

\section{Results}

\subsection{Descriptive Evaluation of Monitoring Data}

In the study period, L. pneumophila was the only species isolated from the water systems of the toilet cabins, with a total of 217 positive samples considering the entire dataset $(54.5 \%, 217 / 398)$. Time trend and differences among serogroups are depicted in Figure 2. The annual variability of L. pneumophila contamination ranged from $30.4 \%$ $(14 / 46)$ in 2013 to $81.6 \%$ (31/36) in 2018. However, when the sampling size increased, approximately half of the samples were contaminated by L. pneumophila, namely $50.6 \%$ $(86 / 170)$ and 53.1\% (43/81) (Figure 2). Among positive samples, 64\% were colonized by L. pneumophila sg $1,27 \%$ by L. pneumophila serogroups 2-14, while mixed Legionella concentration (sg 1 and serogroups 2-14) was obtained in 9\% samples. In positive samples, one-way ANOVA analysis indicated that Legionella concentration did not differ significantly through the 6-year monitoring period (one-way ANOVA, $p=0.32$ ).

On the whole of positive samples (Table 2), the geometric mean of L. pneumophila concentrations was $4.39 \times 10^{3} \pm 4.97 \mathrm{CFU} / \mathrm{L}$. Of the positive samples $(100 / 217), 46 \%$ contained an L. pneumophila concentration between 1001 and 10,000 CFU/L, and the majority of them were L. pneumophila sg $1(61 / 100 ; 61 \%)$. However, L. pneumophila sg 1 was the most frequently isolated serogroup also in the lower (101-1000 CFU/L) and in the higher concentration categories $(\geq 10,001 \mathrm{CFU} / \mathrm{L})$, with an occurrence of $88.6 \%(39 / 44)$ and $55 \%$ $(38 / 69)$, respectively. 


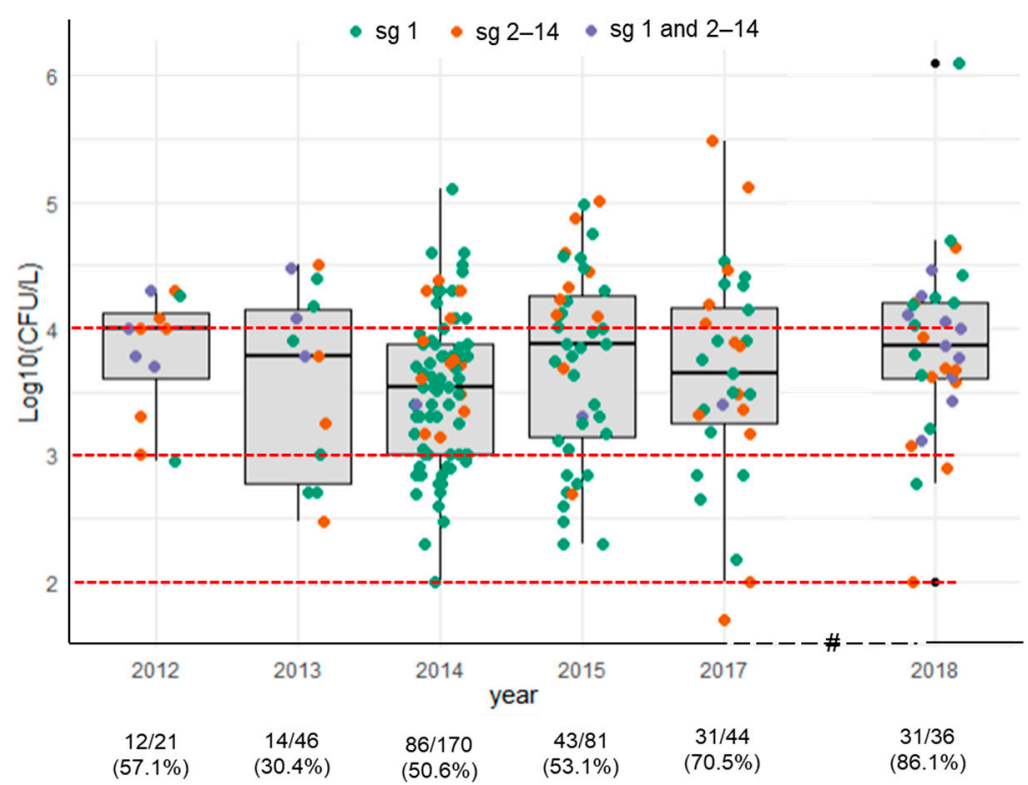

Figure 2. Annual variability of L. pneumophila concentrations in positive samples. For each year, the distribution of the whole Legionella concentration values is represented by boxplot and the serogroups are identified by different colors. Red dashed lines correspond to Legionella thresholds in water for the application of control measures in healthcare and community settings, according to Italian guidelines [23].

Table 2. Contamination of L. pneumophila in positive samples. For load distribution categories, percentages refer to the column (calculation based on the type of serogroup).

\begin{tabular}{|c|c|c|c|c|}
\hline & L. pneumophila Total & L. pneumophila sg1 & $\begin{array}{l}\text { L. pneumophila sg } \\
\text { 2-14 }\end{array}$ & $\begin{array}{l}\text { Mixed L. pneumophila } \\
\text { sg } 1 \text { and sg 2-14 }\end{array}$ \\
\hline Positive samples $\left(\mathrm{n}^{\circ}, \%\right)$ & $217 / 398(54.5 \%)$ & $139 / 217(64.1 \%)$ & $58 / 217(26.7 \%)$ & $20 / 217(9.2 \%)$ \\
\hline \multicolumn{5}{|l|}{ Count (CFU/L) } \\
\hline Geometric mean & $4.93 \times 10^{3} \pm 4.97$ & $3.67 \times 10^{3} \pm 4.96$ & $5.72 \times 10^{3} \pm 5.74$ & $7.00 \times 10^{3} \pm 2.45$ \\
\hline Median & $5.00 \times 10^{3}$ & $4.10 \times 10^{3}$ & $6.68 \times 10^{3}$ & $6.63 \times 10^{3}$ \\
\hline $\operatorname{IQR}\left(1^{\circ}-3^{\circ}\right.$ quartiles $)$ & $1.50 \times 10^{3}-1.30 \times 10^{4}$ & $1.00 \times 10^{3}-1.14 \times 10^{4}$ & $2.13 \times 10^{3}-1.93 \times 10^{4}$ & $3.79 \times 10^{3}-1.22 \times 10^{4}$ \\
\hline \multicolumn{5}{|c|}{ Load distribution categories $\left(\mathrm{n}^{\circ}, \%\right)$} \\
\hline$\leq 100 \mathrm{CFU} / \mathrm{L}$ & $4 / 217(1.8 \%)$ & $1 / 139(0.7 \%)$ & $3 / 58(5.2 \%)$ & $0 / 20(0 \%)$ \\
\hline 101-1000 CFU/L & $44 / 217(20.3 \%)$ & $39 / 139(28.1 \%)$ & $5 / 58(8.6 \%)$ & $0 / 20(0 \%)$ \\
\hline $1001-10,000 \mathrm{CFU} / \mathrm{L}$ & $100 / 217(46.1 \%)$ & $61 / 139(43.9 \%)$ & $27 / 58(46.6 \%)$ & $12 / 20(60 \%)$ \\
\hline$\geq 10,001 \mathrm{CFU} / \mathrm{L}$ & $69 / 217(31.8 \%)$ & $38 / 139(27.3 \%)$ & $23 / 58(39.7 \%)$ & $8 / 20(40 \%)$ \\
\hline
\end{tabular}

\subsection{Fitted Distribution for L. pneumophila Serogroup 1}

In the perspective of QMRA, statistical analysis was focused on L. pneumophila sg 1 (Section 2.3). Since there was no difference in Legionella concentration among different years (ANOVA, $p>0.05$ ), the entire dataset for L. pneumophila sg1 (139 values) was used to derive a theoretical fitted distribution function. Among the tested theoretical distributions, lognormal was the best in fitting the monitoring data considering the AIC as goodness-of-fit criteria, which was the lowest compared to the other distributions. The best estimates of parameters using the MLE method were $\mu=8.166848$ and $\sigma=1.521021$, which represent the mean and the standard deviation of the associated normal distribution. Overall, the quality of the fit for lognormal distribution can be also appreciated using graphic tools (Figure 3), which gave strong evidence that monitoring data were well approximated by the theoretical distribution with the above-mentioned $\mu$ and $\sigma$ parameters. Namely, the distribution of the empirical data overlaps with the lognormal fitted distribution, both in the probability 
density function (PDF) and in the cumulative density function (CDF) plots (Figure 3a,b). Likewise, the empirical dataset of the monitoring data did not deviate from the theoretical dataset from the lognormal fitted distribution, when they are plotted against each other in terms of CDF (probability-probability (P-P) plot) or of quantiles (quantile-quantile (Q-Q) plot) (Figure 3c,d).

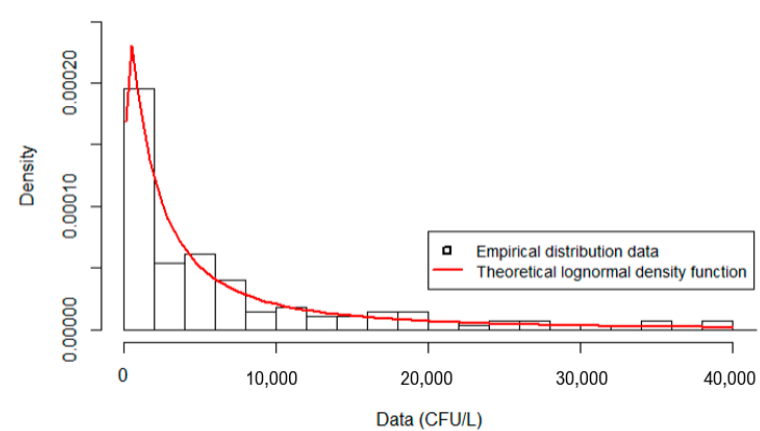

(a)

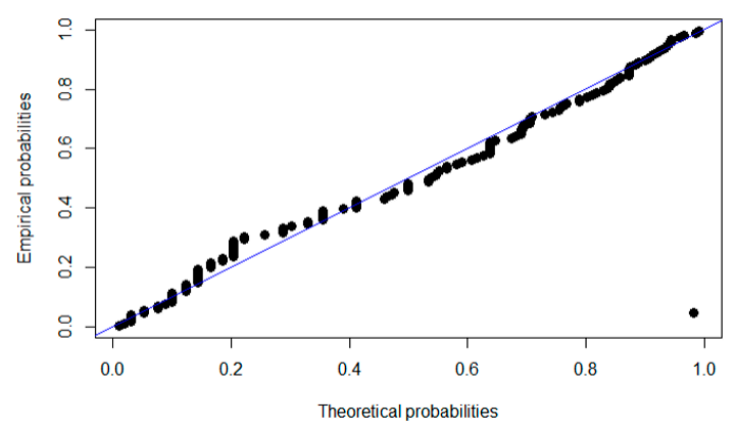

(c)

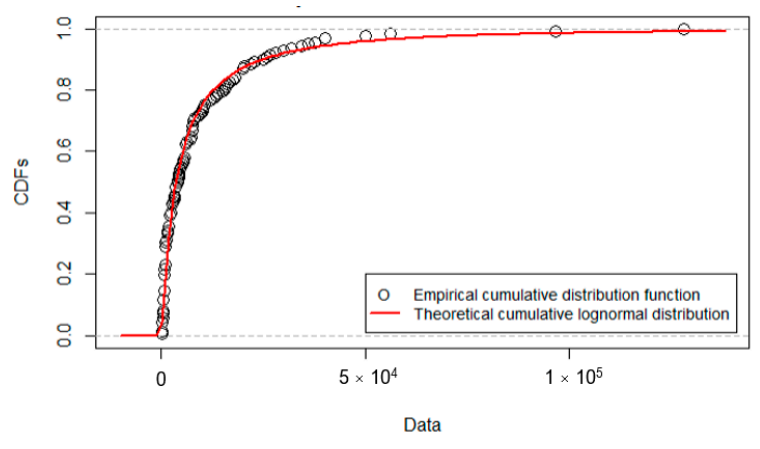

(b)

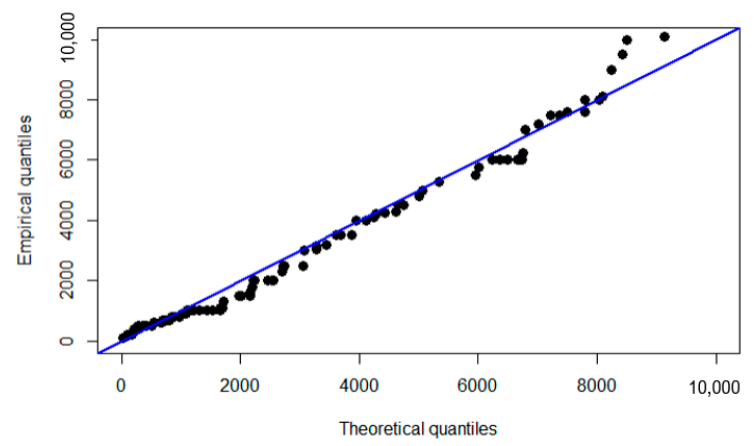

(d)

Figure 3. Results of the fitting of a Lognormal distribution to the monitoring dataset. Empirical and theoretical distributions are represented as probability density function (PDF) (a) or as cumulative density function (CDF) (b). Empirical data set plotted against the theoretical lognormal data set are represented as P-P plot (c) and Q-Q plot (d). A straight blue line represents perfect fitting in (c,d).

\subsection{QMRA Simulation Results}

\subsubsection{Infection Risk from Inhalation of L. pneumophila sg1}

The infection risk was calculated from L. pneumophila sg1 concentration in the water using monitoring data collected from toilet faucet and modeled as a lognormal curve with parameters estimated by best-fitting analysis (see Section 3.2), while the other input parameters were drawn from distributions or estimated as point values, based on literature, as reported in Table 1 . Based on the modeled distributions, the median value of aerosolized L. pneumophila sg1 was $5.40 \times 10^{-3} \mathrm{CFU} / \mathrm{m}^{3}\left(\mathrm{IQR}=4.31 \times 10^{-4}-6.32 \times 10^{-2}\right)$, of inhalation rate was $0.015 \mathrm{~m}^{3} / \mathrm{min}(\mathrm{IQR}=0.014-0.016)$, and of exposure time was $1 \mathrm{~min}(\mathrm{IQR}=0.7-1.3)$. In such exposure scenario, the single use of toilet faucet was responsible for an inhalation dose of L. pneumophila sg1 of $3.86 \times 10^{-5}$ CFU (median value), which corresponded to an infection risk of approximately 2 infections $/ 10^{6}$ exposures, varying in an interquartile range of 2 infections $/ 10^{7}$ exposures and 3 infections $/ 10^{5}$ exposures. Since current guidelines for Legionella prevention are based on bacterial concentrations in water $[19,23]$, we considered the relationship between infection risk and L. pneumophila sg1 load in water collected from the faucets, then we calculated the probability of infection for each concentration category (Figure 4). When L. pneumophila sg1 concentration was $\leq 100 \mathrm{CFU} / \mathrm{L}$, median infection risk was approx. 1 infection $/ 10^{6}$ exposures $\left(\mathrm{IQR}=1.83 \times 10^{-7}-1.97 \times 10^{-6}\right)$, increasing to 11 and 70 infections per $10^{6}$ exposures when Legionella load was 101-1.000 CFU/L and 1001-10,000 CFU/L, respectively. Regarding the highest Legionella threshold for water man- 
agement actions $(\geq 10,001 \mathrm{CFU} / \mathrm{L})$, the median infection risk was approx. 5 infections $/ 10^{4}$ exposures $\left(\mathrm{IQR}=2.23 \times 10^{-4}-2.30 \times 10^{-2}\right)$.

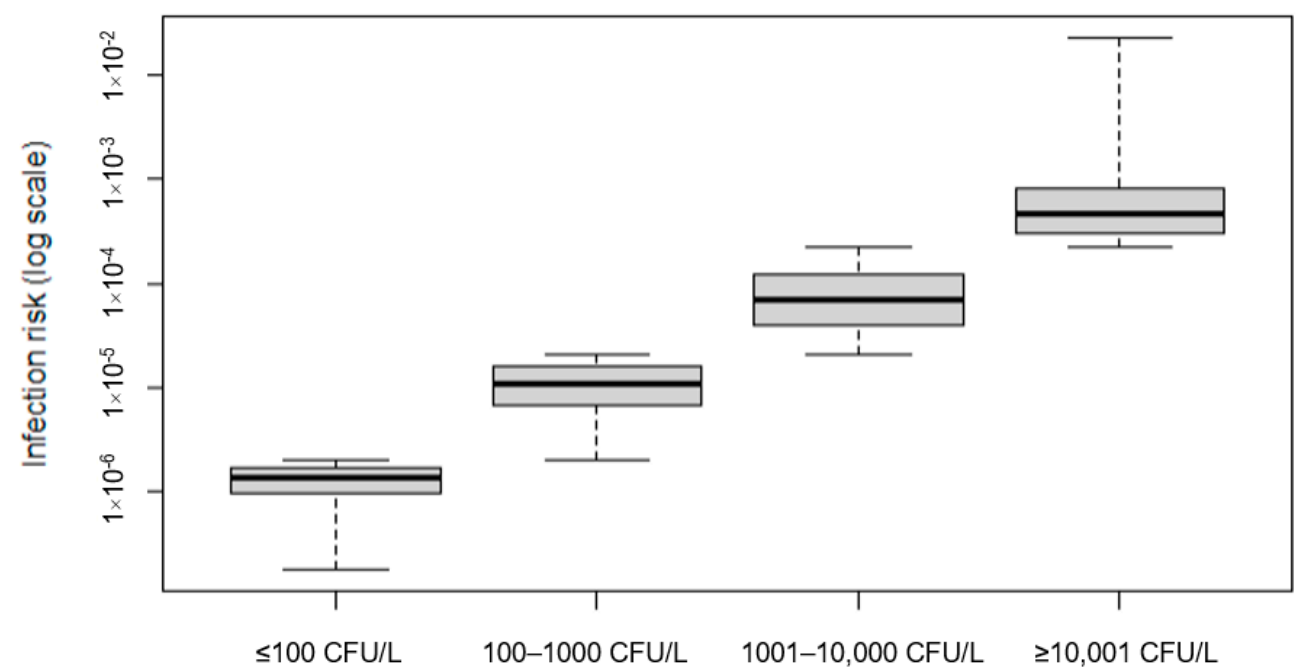

Figure 4. Estimated probability of infection for single use of faucet for different concentrations of $L$. pneumophila sg1 in water, according to concentration categories for water management actions.

\subsubsection{Sensitivity Analysis Results}

For the sensitivity analysis, the fixed values for each input were calculated on an array of 10,000 iterations of their own distribution, as follows: the modal value of L. pneumophila sg1 concentration in water was set at $1.8 \times 10^{4} \mathrm{CFU} / \mathrm{L}$, the mean value of the inhalation rate at $0.015 \mathrm{~m}^{3} / \mathrm{min}$, the mean value of sink use duration at $1 \mathrm{~min}$, and the sink partitioning coefficient was set at the modal value of $1.02 \times 10^{-5} \mathrm{~L} / \mathrm{m}^{3}$. The most impacting parameter was L. pneumophila sg1 concentration followed, in order, by sink partitioning, sink use duration, and inhalation rate (Figure 5). Such results could be attributable to the different ranges of variation of input parameters; for instance, the range of variation of sink use duration (two times its minimum value) was wider than that of the inhalation rate (about $1 / 3$ of the minimum value).

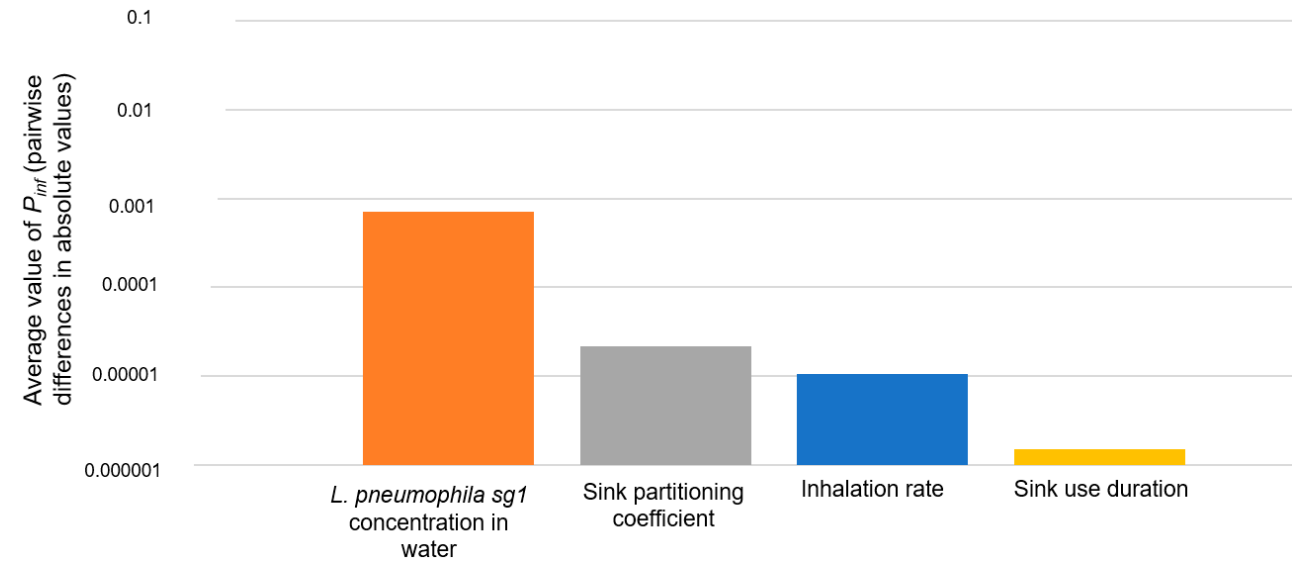

Figure 5. Sensitivity analysis results for the QMRA model. The histograms represent the average values of the pairwise differences of $P_{\text {inf }}$ when all the input parameters are fixed and one varying at a time.

\section{Discussion}

Legionellae are naturally occurring bacteria in any aquatic environment, whose pathogenic potential is mainly attributed to L. pneumophila [34]. From a public health perspective, particular attention is dedicated to L. pneumophila sg 1 since it is the most 
prevalent reported strain of Legionella in building water systems [35] that is currently the predominant serogroup in clinical isolates, accounting for approximately $85 \%$ of cases confirmed by culture worldwide [36-38]. Plumbing systems of LDT can be a source of Legionella, but there is a paucity of environmental surveillance data. At the time of our study, we found only one paper carried out on passenger trains, collecting water from plumbing and toilet water tanks before and after chlorine-based disinfection treatment [22] Our monitoring results showed similar percentages of Legionella positivity, species, and serogroups as in pre-decontamination samples of Quaranta et al. 2012 [22]; namely, they found more than $50 \%$ of water samples positive for Legionella, they identified only the $L$. pneumophila species, and the most frequently isolated was L. pneumophila sg1. Regarding the Legionella concentrations, our study found slightly higher values, but still with a geometric means around $10^{3} \mathrm{CFU} / \mathrm{L}$.

To date, health risk coming from sink exposure is rarely modeled since studies have focused on other residential water uses, mainly having a shower, bathtub, and whirlpool [39-42]. In this paper, we focused on sink exposure since such a scenario could be relevant for the LDT, and monitoring data were available to avoid adapting data collected from the literature. Available L. pneumophila load referred to concentration in water; thus, the dose was calculated using a sink-specific aerosolization ratio because it can vary based on the type of water fixture (i.e., shower, sink, toilet, pool spa, whirlpool), as thoroughly described by Hines et al. (2014) [43]. Although the water provided on LDT is not considered potable, the risk assessment and management for Legionella can be carried out as in the case of drinking water distribution systems (Directive EU 2020/2184), owing to the similarities of the water plant configuration and of critical points for the proliferation. Currently, management actions for Legionella prevention in community or healthcare settings are based on Legionella spp. detection in water collected from the fixtures or collection tanks of the plumbing system. The thresholds for the adoption of control measures usually range from $10^{2}$ to $10^{5} \mathrm{CFU}$ per liter $[18,19]$, with the above-mentioned Directive EU 2020/2184 setting a parametric value of 1000 CFU / L for applying restriction of the use and remedial actions to drinking water distribution systems. However, such values are not established on a risk-based criterion [20], and specific guidelines to prevent Legionella's transmission on LDT are currently missing. Therefore, we performed a quantitative assessment to understand the infection risk associated with Legionella thresholds in water on LDT, using Italian guidelines as an example for deriving concentration categories. The model outcomes showed a very low risk when L. pneumophila sg1 concentration was $\leq 100 \mathrm{CFU} / \mathrm{L}$, with approximately 1 infection per 1 million exposed passengers. However, monitoring results revealed that around $30 \%$ of samples were $\geq 10,000 \mathrm{CFU} / \mathrm{L}$, and, in that case, infection risk increased up to 5 infections per 10,000 exposed (median value). Although such health risk is overall low, the obtained results deserve consideration since public transport is commonly used by populations with risk factors for the development of a severe form of the disease, such as older age, surgery, immunodeficiency, or smoking habit. Moreover, personnel employed on LDT (i.e., train drivers, cleaning staff onboard) could have a prolonged exposure than passengers. This topic is currently little explored, but a seroprevalence study on Turkish bus drivers revealed high L. pneumophila antibody levels among such workers, demonstrating a chronic exposure to the pathogen, probably coming from bus air-conditioning systems [44].

\section{Limitations of the Study}

In this paper, the Legionella risk assessment on LDT was carried out considering the faucets as the only source of Legionella-laden aerosol. Nevertheless, additional exposure could derive also from toilet flushing, which was not modeled owing to the lack of monitoring data. Another limitation of the study could be that illness risk was not calculated, and health risk was expressed as the probability of infection. However, the development of illness can greatly vary depending on the underlying health of individuals, with little understanding of the mechanisms responsible for the development of self-limiting symptoms 
(Pontiac fever) or the severe pneumonia outcome (Legionnaire's disease) [28]. Regarding the trend of Legionella contamination over time, we considered together L. pneumophila sg1 values coming from various annual monitoring campaigns for statistical fitting of data. Nevertheless, we found an annual variability in serogroups occurrence that was not further addressed since it goes beyond the aim of the present study. Therefore, the analysis of time trend of L. pneumophila contamination could be further investigated considering not only sampling size for each investigated period but also variables, both technical (e.g., renewal of trains, disinfection systems) and environmental (e.g., climate) ones.

\section{Conclusions}

This paper addressed a currently little-explored topic represented by the risk assessment of Legionella on LDT, using monitoring data collected from sinks of toilet cabins. Monitoring results on faucets showed that water systems on LDT can be colonized by Legionella, which was responsible for a low number of infections acquired through the studied exposure scenario. Nevertheless, some samples showed a very high bacterial load, which in turn was associated with an increase in the infection risk. Overall, this paper highlighted the importance of Legionella risk assessment also on LDT, which could be useful for planning adequate control measures to protect human health, both of passengers and of workers.

Author Contributions: Conceptualization, A.C. and I.F.; methodology, A.C. and I.F.; software, I.F. and L.C.; formal analysis, I.F. and L.C.; investigation, O.D.G., G.D., F.T., F.A., M.D., M.T.M., I.F., A.C., M.V., L.C.; writing — original draft preparation, I.F. and A.C.; writing-review and editing, I.F., O.D.G., G.D., F.T., F.A., M.D., L.C., M.V., M.T.M., A.C.; supervision, A.C., M.T.M. All authors have read and agreed to the published version of the manuscript.

Funding: This research received no external funding.

Institutional Review Board Statement: Not applicable.

Informed Consent Statement: Not applicable.

Data Availability Statement: Data available on request due to privacy restrictions.

Conflicts of Interest: The authors declare no conflict of interest.

\section{References}

1. WHO. Health Aspects of Plumbing; World Health Organization, World Council of Plumbing, Eds.; World Health Organization (WHO): Geneva, Switzerland, 2006; ISBN 9241563184. Available online: https://www.who.int/publications/i/item/9241563184 (accessed on 1 December 2021).

2. Falkinham, J.O.; Pruden, A.; Edwards, M. Opportunistic Premise Plumbing Pathogens: Increasingly Important Pathogens in Drinking Water. Pathogens 2015, 4, 373-386. [CrossRef]

3. Falkinham, J.O., 3rd. Living with Legionella and Other Waterborne Pathogens. Microorganisms 2020, 8, 2026. [CrossRef]

4. $\quad$ Benedict, K.M.; Reses, H.; Vigar, M.; Roth, D.M.; Roberts, V.A.; Mattioli, M.; Cooley, L.A.; Hilborn, E.D.; Wade, T.J.; Fullerton, K.E.; et al. Surveillance for waterborne disease outbreaks associated with drinking water-United States, $2013-2014$. MMWR Morb. Mortal Wkly. Rep. 2017, 66, 1216-1221. [CrossRef] [PubMed]

5. Hamilton, K.A.; Prussin, A.J., 2nd; Ahmed, W.; Haas, C.N. Outbreaks of Legionnaires' Disease and Pontiac Fever 2006-2017. Curr. Environ. Health Rep. 2018, 5, 263-271. [CrossRef] [PubMed]

6. $\quad$ ECDC. Legionnaires' Disease. In Annual Epidemiological Report for 2019; European Centre for Disease Prevention and Control (ECDC): Stockholm, Sweden, 2021; Available online: https:/ /www.ecdc.europa.eu/en/publications-data/legionnaires-diseaseannual-epidemiological-report-2019 (accessed on 1 December 2021).

7. Bauer, M.; Mathieu, L.; Deloge-Abarkan, M.; Remen, T.; Tossa, P.; Hartemann, P.; Zmirou-Navier, D. Legionella bacteria in shower aerosols increase the risk of Pontiac fever among older people in retirement homes. J. Epidemiol. Community Health 2008, 62, 913-920. [CrossRef] [PubMed]

8. $\quad$ Breiman, R.F.; Fields, B.S.; Sanden, G.N.; Volmer, L.; Meier, A.; Spika, J.S. Association of shower use with Legionnaires' disease. Possible role of amoebae. JAMA 1990, 263, 2924-2926. [CrossRef] [PubMed]

9. De Giglio, O.; Fasano, F.; Diella, G.; Lopuzzo, M.; Napoli, C.; Apollonio, F.; Brigida, S.; Calia, C.; Campanale, C.; Marzella, A.; et al. Legionella and legionellosis in touristic-recreational facilities: Influence of climate factors and geostatistical analysis in Southern Italy (2001-2017). Environ. Res. 2019, 178, 108721. [CrossRef] 
10. Katsiaflaka, A.; Pournaras, S.; Kristo, I.; Mouchtouri, V.A.; Kyritsi, M.; Velonakis, E.; Vatopoulos, A.C.; Hadjichristodoulou, C. Epidemiological investigation of Legionella pneumophila serogroup 2 to 14 isolates from water samples by amplified fragment length polymorphism and sequence-based typing and detection of virulence traits. Appl. Environ. Microbiol. 2016, 82, 6102-6108. [CrossRef]

11. Azara, A.; Piana, A.; Sotgiu, G.; Dettori, M.; Deriu, M.G.; Masia, M.D.; Are, B.M.; Muresu, E. Prevalence study of Legionella spp. contamination in ferries and cruise ships. BMC Public Health 2006, 6, 100. [CrossRef]

12. Laganà, P.; Gambuzza, M.E.; Delia, S. Legionella risk assessment in cruise ships and ferries. Ann. Agric. Environ. Med. 2017, 24, 276-282. [CrossRef]

13. De Filippis, P.; Mozzetti, C.; Messina, A.; D'Alò, G.L. Prevalence of Legionella in retirement homes and group homes water distribution systems. Sci. Total Environ. 2018, 643, 715-724. [CrossRef]

14. Borella, P.; Montagna, M.T.; Romano-Spica, V.; Stampi, S.; Stancanelli, G.; Triassi, M.; Neglia, R.; Marchesi, I.; Fantuzzi, G.; Tatò, D.; et al. Legionella infection risk from domestic hot water. Emerg. Infect. Dis. 2004, 10, 457-464. [CrossRef]

15. Mathys, W.; Stanke, J.; Harmuth, M.; Junge-Mathys, E. Occurrence of Legionella in hot water systems of single-family residences in suburbs of two German cities with special reference to solar and district heating. Int. J. Hyg. Environ. Health 2008, 211, 179-185. [CrossRef]

16. Donohue, M.J.; King, D.; Pfaller, S.; Mistry, J.H. The sporadic nature of Legionella pneumophila, Legionella pneumophila Sg1 and Mycobacterium avium occurrence within residences and office buildings across 36 states in the United States. J. Appl. Microbiol. 2019, 126, 1568-1579. [CrossRef]

17. Bonetta, S.; Bonetta, S.; Ferretti, E.; Balocco, F.; Carraro, E. Evaluation of Legionella pneumophila contamination in Italian hotel water systems by quantitative real-time PCR and culture methods. J. Appl. Microbiol. 2011, 108, 1576-1583. [CrossRef] [PubMed]

18. WHO. Legionella and the Prevention of Legionellosis; World Health Organization (WHO): Geneva, Switzerland, 2007; ISBN 9241562978. Available online: https:/ / apps.who.int/iris/handle/10665/43233 (accessed on 1 December 2021).

19. ESGLI. European Technical Guidelines for the Prevention, Control and Investigation of Infections Caused by Legionella Species; European Society of Clinical Microbiology and Infectious Diseases Study Group for Legionella Infections (ESGLI): Basel, Switzerland, 2017; Available online: https://www.ecdc.europa.eu/en/publications-data/european-technical-guidelines-prevention-control-andinvestigation-infections (accessed on 1 December 2021).

20. Hamilton, K.A.; Hamilton, M.T.; Johnson, W.; Jjemba, P.; Bukhari, Z.; LeChevallier, M.; Haas, C.N.; Gurian, P.L. Risk-Based Critical Concentrations of Legionella pneumophila for Indoor Residential Water Uses. Environ. Sci. Technol. 2019, 53, 4528-4541. [CrossRef] [PubMed]

21. WHO. Quantitative Microbial Risk Assessment: Application for Water Safety Management; World Health Organization (WHO): Geneva, Switzerland, 2016.

22. Quaranta, G.; Vincenti, S.; Ferriero, A.M.; Boninti, F.; Sezzatini, R.; Turnaturi, C.; Gliubizzi, M.D.; Munafò, E.; Ceccarelli, G.; Causarano, C.; et al. Legionella on board trains: Effectiveness of environmental surveillance and decontamination. BMC Public Health 2012, 12, 618. [CrossRef] [PubMed]

23. Italian Ministry of Health. Guidelines for the Prevention and Control of Legionellosis. 2015. Available online: https://www. salute.gov.it/portale/documentazione/p6_2_2_1.jsp?id=2362 (accessed on 1 December 2021). (In Italian)

24. R Core Team. R: A Language and Environment for Statistical Computing; R Foundation for Statistical Computing: Vienna, Austria, 2018; Available online: www.Rproject.org (accessed on 1 December 2021).

25. Pouillot, R.; Delignette-Muller, M.L. Evaluating variability and uncertainty in microbial quantitative risk assessment using two R packages. Int. J. Food Microbiol. 2010, 142, 330-340. [CrossRef]

26. Gan, F.F.; Koehler, K.J. Goodness-of-Fit Tests Based on P-P Probability Plots. Technometrics 1990, 32, 289-303. [CrossRef]

27. Haas, C.; Rose, J.; Gerba, C. Risk assessment paradigms. In Quantitative Microbial Risk Assessment, 2nd ed.; John Wiley \& Sons, Inc.: Hoboken, NJ, USA, 2014; ISBN 9781118145296.

28. Whiley, H.; Keegan, A.; Fallowfield, H.; Ross, K. Uncertainties associated with assessing the public health risk from Legionella. Front. Microbiol. 2014, 5, 501. [CrossRef]

29. Hamilton, K.A.; Haas, C.N. Critical review of mathematical approaches for quantitative microbial risk assessment (QMRA) of Legionella in engineered water systems: Research gaps and a new framework. Environ. Sci. Water Res. Technol. 2016, 2, 599-613. [CrossRef]

30. Bollin, G.E.; Plouffe, J.F.; Para, M.F.; Hackman, B. Aerosols containing Legionella pneumophila generated by shower heads and hot-water faucets. Appl. Environ. Microbiol. 1995, 50, 1128-1131. [CrossRef]

31. U.S. Environmental Protection Agency. Exposure Factors Handbook: Chapter 6-Inhalation Rates. 2011. Available online: https:/ / cfpub.epa.gov/ncea/risk/recordisplay.cfm?deid=236252 (accessed on 1 December 2021).

32. Armstrong, T.W.; Haas, C.N. A quantitative microbial risk assessment model for Legionnaires' disease: Animal model selection and dose-response modeling. Risk Anal. 2007, 27, 1581-1596. [CrossRef] [PubMed]

33. Federigi, I.; Bonadonna, L.; Bonanno Ferraro, G.; Briancesco, R.; Cioni, L.; Coccia, A.M.; Della Libera, S.; Ferretti, E.; Gramaccioni, L.; Iaconelli, M.; et al. Quantitative Microbial Risk Assessment as support for bathing water profiling. Mar. Pollut. Bull. 2020, 157, 111318. [CrossRef]

34. Ratcliff, R.M.; Lanser, J.A.; Manning, P.A.; Heuzenroeder, M.W. Sequence-based classification scheme for the genus Legionella targeting the mip gene. J. Clin. Microbiol. 1998, 36, 1560-1567. [CrossRef] [PubMed] 
35. National Academies of Sciences, Engineering, and Medicine. Management of Legionella in Water Systems; The National Academies Press: Washington, DC, USA, 2020. [CrossRef]

36. Yu, V.L.; Plouffe, J.F.; Pastoris, M.C.; Stout, J.E.; Schousboe, M.; Widmer, A.; Summersgill, J.; File, T.; Heath, C.M.; Paterson, D.L.; et al. Distribution of Legionella species and serogroups isolated by culture in patients with sporadic communityacquired legionellosis: An international collaborative survey. J. Infect. Dis. 2002, 186, 127-128. [CrossRef] [PubMed]

37. Fontana, S.; Scaturro, M.; Rota, M.C.; Caporali, M.G.; Ricci, M.L. Molecular typing of Legionella pneumophila serogroup 1 clinical strains isolated in Italy. Int. J. Med. Microbiol. 2014, 304, 597-602. [CrossRef]

38. Beauté, J. Legionnaires' disease in Europe, 2011 to 2015. European Legionnaires' Disease Surveillance Network. Eurosurveillance 2017, 22, 30566. [CrossRef]

39. Schoen, M.E.; Ashbolt, N.J. An in-premise model for Legionella exposure during showering events. Water Res. 2011, 45, 5826-5836. [CrossRef]

40. Azuma, K.; Uchiyama, I.; Okumura, J. Assessing the risk of Legionnaires' disease: The inhalation exposure model and the estimated risk in residential bathrooms. Regul. Toxicol. Pharmacol. 2013, 65, 1-6. [CrossRef]

41. Bouwknegt, M.; Schijven, J.F.; Schalk, J.A.; de Roda Husman, A.M. Quantitative risk estimation for a Legionella pneumophila infection due to whirlpool use. Risk Anal. 2013, 33, 1228-1236. [CrossRef] [PubMed]

42. Blanky, M.; Sharaby, Y.; Rodríguez-Martínez, S.; Halpern, M.; Friedler, E. Greywater reuse-Assessment of the health risk induced by Legionella pneumophila. Water Res. 2017, 125, 410-417. [CrossRef]

43. Hines, S.A.; Chappie, D.J.; Lordo, R.A.; Miller, B.D.; Janke, R.J.; Lindquist, H.A.; Fox, K.R.; Ernst, H.S.; Taft, S.C. Assessment of relative potential for Legionella species or surrogates inhalation exposure from common water uses. Water Res. 2014, 56, 203-213. [CrossRef] [PubMed]

44. Polat, Y.; Ergin, C.; Kaleli, I.; Pinar, A. Riskli bir meslek olarak profesyonel uzun yol süruocolerinde Legionella pneumophila seropozitifldginin araştirilmasi [Investigation of Legionella pneumophila seropositivity in the professional long distance drivers as a risky occupation]. Mikrobiyol. Bul. 2007, 41, 211-217. (In Turkish) [PubMed] 